\title{
Food and Beverage Ingredients Induce Formation of Silver Nanoparticles in Products Stored within Nanotechnology-Enabled Packaging
}

\author{
Tianxi Yang', Teena Paulose ${ }^{2}$, Benjamin W. Redan', James C. Mabon ${ }^{3}$, Timothy V. Duncan ${ }^{1 *}$ \\ ${ }^{1}$ Center for Food Safety and Nutrition, United States Food and Drug Administration, Bedford Park IL, \\ 60501 \\ ${ }^{2}$ Department of Food Science and Nutrition, Illinois Institute of Technology, Bedford Park IL, 60501 \\ ${ }^{3}$ Materials Research Laboratory, University of Illinois at Urbana-Champaign, Urbana, Illinois 61801, USA \\ *Corresponding author: timothy.duncan@fda.hhs.gov
}

\section{SUPPORTING INFORMATION SECTION}

\section{Supplementary Materials and Methods}

Full ingredient lists of all commercial foods and beverages used in this study, as listed on product labels. Ingredients are listed in order of decreasing concentration. Sprite drink (Coca-Cola Company) contains carbonated water, high fructose corn syrup, citric acid, natural favors, sodium citrate and sodium benzoate. Squirt drink (Keurig Dr Pepper) contains carbonated water, high fructose corn syrup, grapefruit concentrate, natural favors, citric acid, modified corn starch, ester gum, sodium benzoate and calcium disodium EDTA. White Cranberry Juice (Ocean Spray) contains filtered water, sugar, white grape juice (water, white grape juice concentrate), cranberry juice (water, cranberry juice concentrate), citric acid, ascorbic acid, natural flavor, sodium citrate. Whole milk (Gossner Foods) contains milk, protein, saturated fat, vitamin A, vitamin D3, cholesterol, sodium, potassium, calcium and sugars. 2\% fat milk (Gossner Foods) contains reduced fat milk, protein, saturated fat, vitamin A, vitamin D3, cholesterol, sodium, potassium, calcium, palmitate and sugars. Fat free milk (Gossner Foods) contains skim milk, protein, vitamin A, vitamin D3, cholesterol, sodium, potassium, calcium, palmitate and sugars. Non-fat Greek yogurt (Chobani $\left.{ }^{\circledR}\right)$ contains cultured nonfat milk, peaches, cane sugar, water, fruit pectin, natural flavors, locust bean gum, lemon juice concentrate. All-purpose flour (Gold Metal ${ }^{\mathrm{TM}}$ ) contains bleached wheat flour, niacin (a B vitamin), iron, thiamine mononitrate (vitamin B1), riboflavin (vitamin B2), enzymes, folic acid (a B vitamin), wheat and gluten.

Scanning Transmission Electron Microscopy (STEM): STEM samples of AgNPs were prepared by drop casting a suspension of AgNPs on a 200 mesh C-coated $\mathrm{Cu}$ grid (Ted Pella, Redding, CA). Microscopy of AgNPs formed in food ingredients solutions and commercial beverages (as shown in Figure 2, Figure S1, Figure S3 and Figures S5-S6) was performed at the University of Illinois at Urbana- 
Champaign (Urbana, Illinois) in the Materials Research Laboratory. STEM images of AgNPs were acquired on a JEOL 2200FS STEM with CEOS CESCOR probe spherical aberration corrector. It was operated at $200 \mathrm{kV}$ and all the images are dark field STEM (Scanning TEM). The lowest magnification images are a low mag mode. All the others are HAADF (High Angle Annular Dark Field) STEM. Microscopy of AgNPs used for manufacturing PNCs (as shown in Figure 4A) was performed on a Hitachi HD-2300 cFEG STEM using bright field TEM mode at $200 \mathrm{keV}$ at Northwestern University (Evanston, IL) in the NUANCE facility.

Film Digestion: For the determination of total Ag amounts incorporated in polymers, films were first digested with a CEM (Matthews, NC) Discover SP-D microwave digester. Approximately $200 \mathrm{mg}$ film, $10 \mathrm{~mL}$ concentrated $\mathrm{HNO}_{3}$ and a magnetic bead were added in a quartz vessel. Digestion was performed by heating the quartz vessel to $220{ }^{\circ} \mathrm{C}$ with a ramp time of $4 \mathrm{~min}$ and a hold time of $7 \mathrm{~min}$. Maximum pressure and maximum power were set at 400 psi and $300 \mathrm{~W}$, respectively. Samples were cooled and diluted with nanopure water before ICP-MS analysis.

Inductively Coupled Plasma Mass Spectrometry (ICP-MS): ICP-MS was performed on an Agilent (Santa Clara, CA) 8800 triple quadrupole ICP-MS with ASX-500 series autosampler. For ICP-MS analysis of total $\mathrm{Ag}$ amounts in polymers, digestion samples were diluted to $2 \% \mathrm{HNO}_{3}$. For ICP-MS analysis of total released $\mathrm{Ag}$, release samples in sachets were acidified to $2 \% \mathrm{HNO}_{3}$. ICP-MS standard solutions were prepared by diluting $1000 \mu \mathrm{g} / \mathrm{mL}$ of Ag ions standards purchased from SCP Science. Single quadrupole MS mode was used for ICP-MS analysis. RF power was set at $1550 \mathrm{~W}$ and RF matching was set at $1.8 \mathrm{~V}$. Carrier gas flow was $0.95 \mathrm{~L} / \mathrm{min}$. Lenses were autotuned using $1 \mu \mathrm{g} / \mathrm{L}$ tuning solution (Agilent). Sample uptake time was 30 seconds with $0.5 \mathrm{rpm}$ nebulizer pump speed and 40 seconds stabilization. Three rinse cycles were run between samples with $2 \% \mathrm{HNO}_{3}$. The internal standard was $200 \mu \mathrm{g} / \mathrm{L} \mathrm{Rh}$, diluted from $1000 \mu \mathrm{g} / \mathrm{mL}$ internal standard mix purchased from Agilent. SP-ICP-MS analyses of AgNPs were performed in time-resolved analysis (TRA) mode using an integration time of $3 \mathrm{~ms}$ per point. The sample flow rate to the nebulizer was $0.346 \mathrm{~mL} / \mathrm{min}$. AgNP reference material $(50 \mathrm{~nm}, 5 \mathrm{ng} / \mathrm{L}$ ) were diluted from commercial AgNPs $(50 \mathrm{~nm}, 0.02 \mathrm{mg} / \mathrm{mL})$ purchased from nanoComposix. Inc. Sample uptake time was 120 seconds with $0.1 \mathrm{rpm}$ nebulizer pump speed and 30 seconds stabilization. Three rinse cycles were run between samples with nanopure water. Data analysis was performed using ICP-MS MassHunter software. Particle baseline and detection threshold were automatically determined in the MassHunter software.

UV-Visible Spectroscopy: UV-Visible spectroscopy was conducted on a PerkinElmer Lambda 35 spectrometer using a polystyrene or quartz cuvette. Typical scan speed was $480 \mathrm{~nm} / \mathrm{min}$. 


\section{Supplementary Results and Discussion}

Factors that influence formation of AgNPs in foods and beverages. The effect of reducing agent concentration on AgNP formation in foods and beverage was probed by recording visual changes to aqueous solutions of two reducing ingredients (maltodextrin and vitamin $\mathrm{C}$ ) at two different reducing ingredient concentrations after introduction of $\mathrm{AgNO}_{3}$ at $5.8 \mathrm{mM}$ final concentration (Figure S7A). The high and low concentrations of maltodextrin were $9 \mathrm{wt} \%$ and $0.45 \mathrm{wt} \%$, respectively, and the high and low concentrations of vitamin $\mathrm{C}$ were $9 \mathrm{wt} \%$ and $0.03 \mathrm{wt} \%$, respectively. The solutions were stored at $40{ }^{\circ} \mathrm{C}$ and monitored over a period of 21 days. Consistent with our expectations, for both ingredients we observed a positive correlation between the ingredient concentration and the intensity of the solution color, suggesting that a higher concentration of reducing agent resulted in formation of more AgNPs. We note that the low concentration condition for maltodextrin still resulted in AgNP color appearance at the earliest time point. Interestingly, at low maltodextrin concentrations, the color intensity appears to decrease over time, whereas at high concentration, the intensity increases. This may indicate that high concentrations of reducing ingredients can sustain AgNP seeding and growth for longer periods of time and/or can more effectively stabilize the formed AgNPs by acting as capping agents. At low concentrations, the lack of available reducing ingredients for surface stabilization could result in low stability of formed AgNPs and rapid re-oxidation and dissolution. The effect of reductant concentration on the formed AgNP properties reported here is consistent with prior studies that have demonstrated the strong effect of reductant concentration on noble metal nanoparticle formation kinetics and morphology. ${ }^{1}$

Storage temperature and light exposure were also found to impact AgNP formation in the presence of food and beverage ingredients with reducing properties. Figure S7B shows photographs of aqueous solutions of these ingredients, as well as commercial beverages, acquired 24 hours after addition of $\mathrm{AgNO}_{3}$ and storage at either $20^{\circ} \mathrm{C}$ or $40{ }^{\circ} \mathrm{C}$. Figure S7C shows similar samples stored at $40{ }^{\circ} \mathrm{C}$ under ambient room light or in the dark. The effect of light exposure was viewed as important to study because light is known to affect $\mathrm{AgNP}$ formation ${ }^{2}$ and dissolution ${ }^{3}$, particularly in the presence of photoactive organic substances ${ }^{4,5}$, and foods and beverages are often stored in clear containers on illuminated grocery store shelves or alternatively in dark household pantries. During assessment of temperature, we found that the colors of the solutions and beverages stored at $20^{\circ} \mathrm{C}$ differed in intensity and hue compared to those stored at $40{ }^{\circ} \mathrm{C}$, indicating that temperature likely affects both the AgNP formation rate and morphology. (Note that the use of $20^{\circ} \mathrm{C}$ and $40^{\circ} \mathrm{C}$ to represent long term food storage under refrigerated/frozen conditions and room temperature, conditions, respectively, is based on FDA recommendations for assessment of migration of food contact substances. (Please see the Materials and Methods section in the primary article document for more information.) Note that some samples (e.g. milk) were darker in color under the higher temperature condition, whereas others (white cranberry juice) exhibited an opposite effect, signifying that higher storage 
temperatures may favor either AgNP formation or dissolution, depending on the formulation. This variability is also played out in the experiment to assess the impact of light exposure: AgNP concentration, as indicated by solution color intensity, was increased by light in some samples (sucrose solution, Sprite) and diminished in others (Squirt, Domino table sugar solution), although AgNP formation generally seemed to be more favored when samples were exposed to light, consistent with previous work that showed that ambient light may enhance rates of AgNP formation, particularly in the presence of photoactive organic matter. ${ }^{4} 5$ We highlight that with the exception of sucrose solution, all samples showed signs of AgNP formation under the lower temperature condition after only 24 hours, as indicated by SPR peaks in the corresponding UV-Visible spectra (Figure S7D), and even in the dark, most samples exhibited AgNPs. These findings imply that food and beverage ingredients with reducing properties can stimulate conversion of $\mathrm{Ag}^{+}$to diverse forms of AgNPs even during refrigeration and regardless of the lighting conditions.

Finally, we assessed whether AgNP formation by reducing ingredients is influenced by other food and beverage components that do not directly participate $\mathrm{in}^{\mathrm{Ag}^{+}}$reduction. As a test case, we examined three varieties of commercially packaged milk to determine whether fat content would influence formed AgNP quantity or content. This case was deemed relevant because silver-enabled materials have been explored as next generation antimicrobial dairy packaging ${ }^{6,7}$ and fatty acids in dairy products have been reported as effective stabilizers of AgNPs. ${ }^{8,9}$ After spiking milk samples with $\mathrm{AgNO}_{3}$ and storing them for 3 days at $20{ }^{\circ} \mathrm{C}$, we observed more intense color in fat free milk than $2 \%$ fat or whole milk (data not shown). These visual observations were consistent with UV-Vis spectra (Figure S7E), which showed that the SPR peak intensity was inversely correlated to milk fat content. In addition, as the milk fat $\%$ increased, the SPR peak maxima shifted toward longer wavelengths, implying an inverse correlation between fat content and AgNP size. ${ }^{8}$ Since homogenized milk is an emulsion of fat globules stabilized by milk proteins (caseins), ${ }^{10}$ we hypothesize that after they are formed, fatty acid-stabilized AgNPs may become trapped in the lipid phase, with growth to larger sizes supported by lipid-AgNP interactions and further $\mathrm{Ag}^{+}$reduction and deposition on AgNP surfaces occurring at the colloidal interfaces; in effect, the system may behave like a natural version of a microemulsion nanoparticle synthesis engine. ${ }^{11}$ Additionally, the fat globules could possibly interfere with diffusion of $\mathrm{Ag}$ ions and reducing agents, slowing growth and restricting AgNP size. ${ }^{12}$ In contrast, AgNPs in fat free milk could be formed by fast nucleation and effective stabilization by milk proteins that prevented aggregation, which would result in smaller AgNP size and higher AgNP concentration. ${ }^{13,14}$ It is important to note that these explanations for the observed differences in AgNP formation as a function of milkfat content are speculations based on the cited literature. Further experiments will be needed in order to fully explain these data. Nevertheless, this analysis of the effect of milkfat content demonstrates that naturally present or intentionally added non-reducing components can alter morphology and/or amount of AgNPs formed in foods and beverages when $\mathrm{Ag}^{+}$is present, which has obvious 
implications for the fate of silver leached from AgNP-enabled packaging stored in contact with complex food and beverage systems.

\section{Supplementary Figures:}

\section{A}
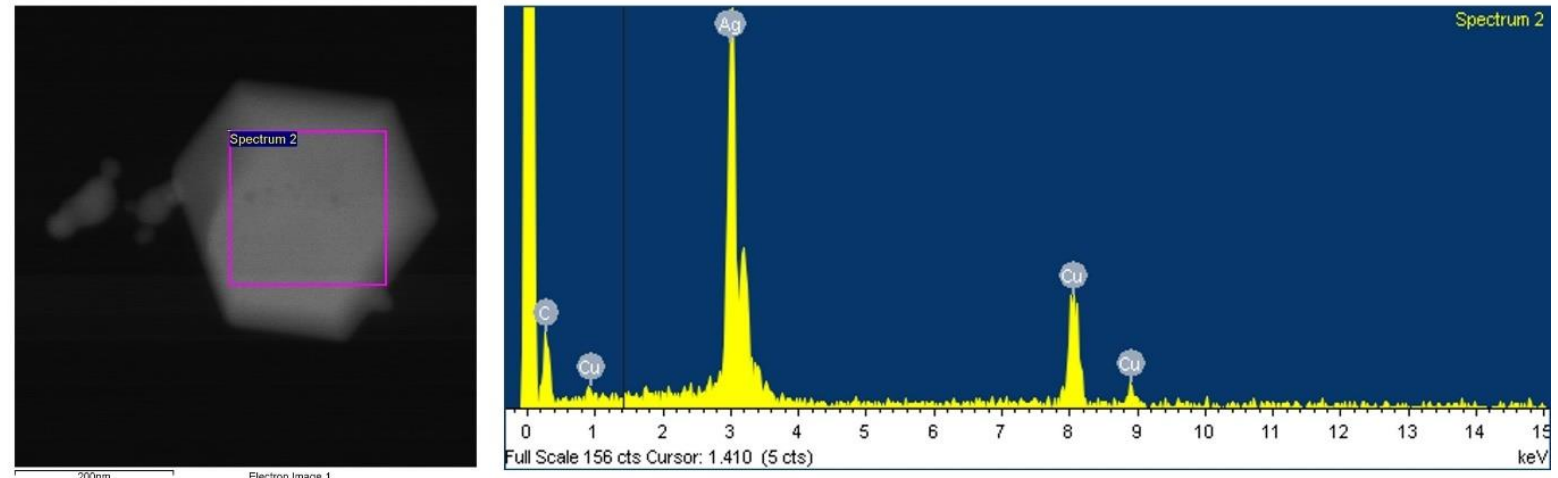

B
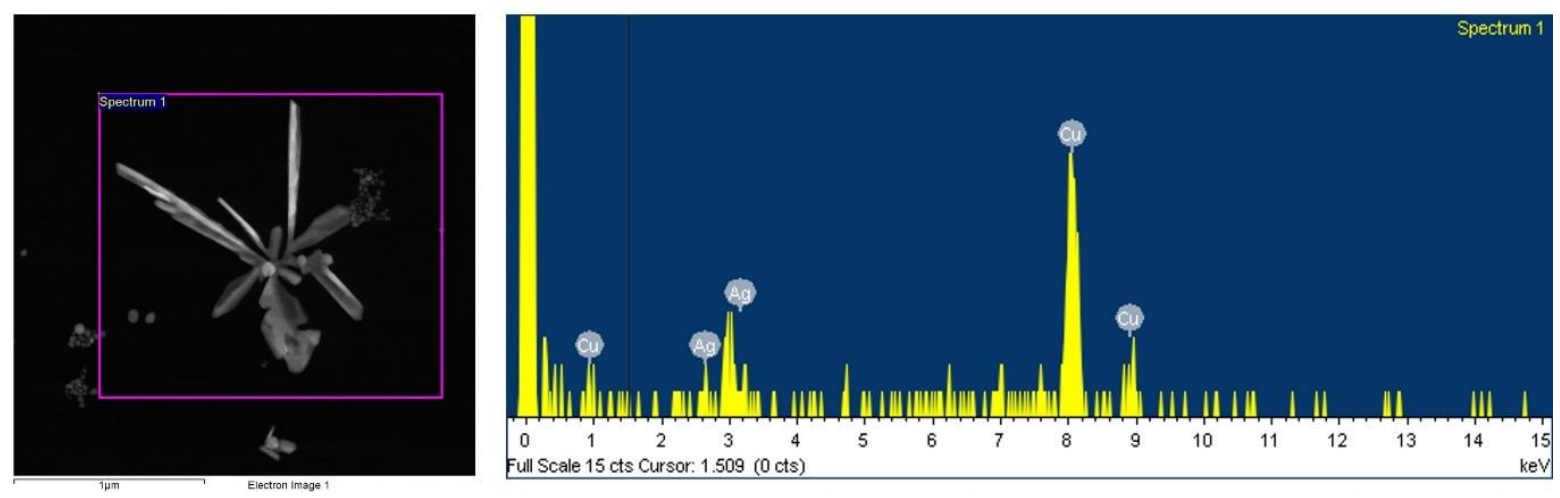

Figure S1. Representative EDS spectra of AgNPs formed in (A) Domino table sugar solution and (B) white cranberry juice with $\mathrm{Ag}^{+}$concentration at $5.8 \mathrm{mM}$ after 3 hours introduction of $\mathrm{Ag}^{+}$. 

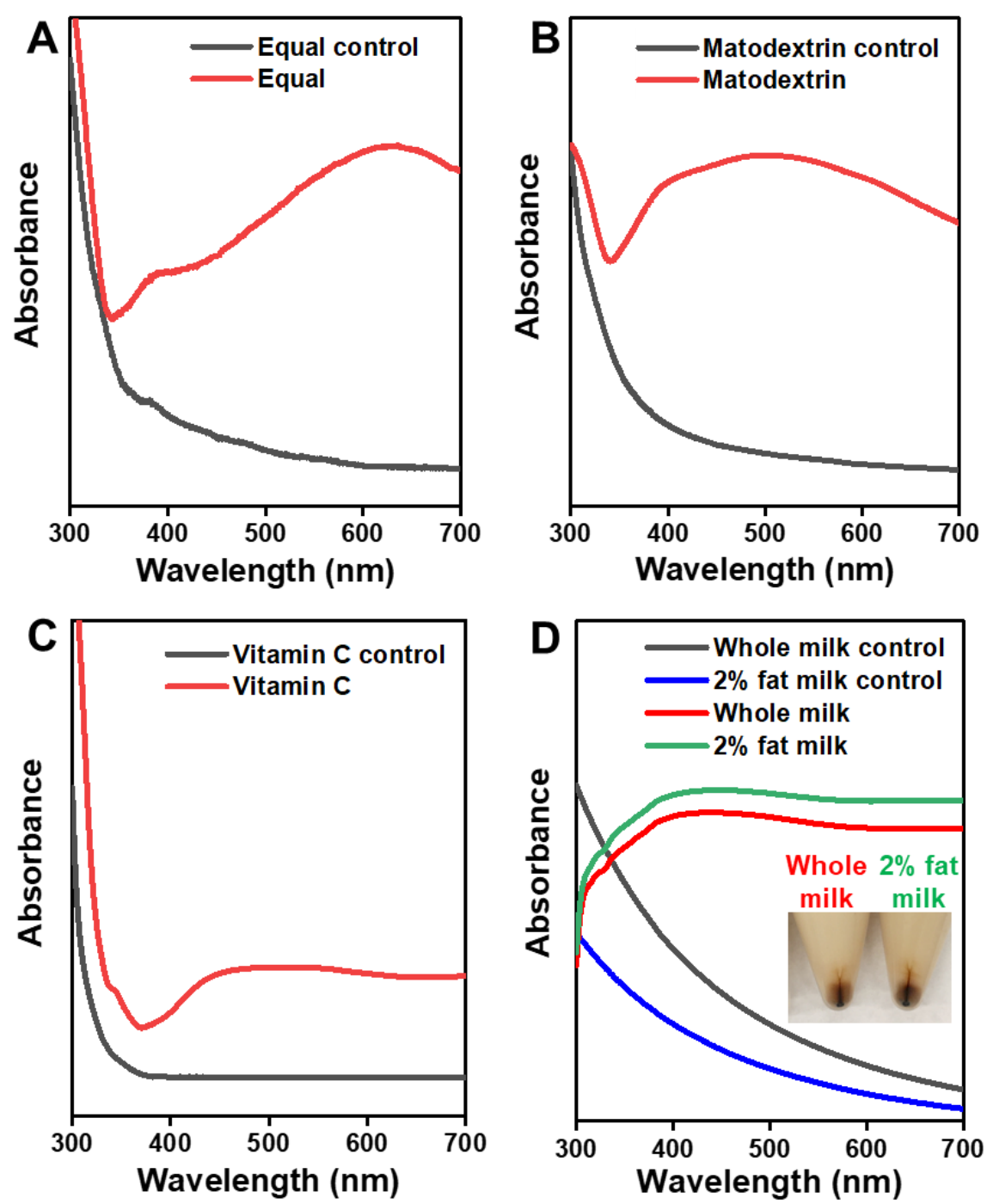

Figure S2. Normalized UV-Vis spectra of $9 \mathrm{wt} \%$ aqueous solutions of reducing food ingredients and milk. (A) $9 \mathrm{wt} \%$ Equal, (B) $0.45 \mathrm{wt} \%$ maltodextrin and (C) $0.03 \mathrm{wt} \%$ vitamin $\mathrm{C}$ as well as (D) whole and low (2\%) fat milk after introduction of $5.8 \mathrm{mM} \mathrm{AgNO}_{3}$. The controls were identical solutions and beverages without $\mathrm{Ag}^{+}$added. Food ingredient solutions were stored for 24 hours and milks were stored for 3 days. The storage temperature was $40{ }^{\circ} \mathrm{C}$. Note that AgNPs formed in milk samples were purified by centrifugation and re-dispersed in water before collecting spectra. The inset shows concentrated AgNPs via centrifugation in milk samples. 

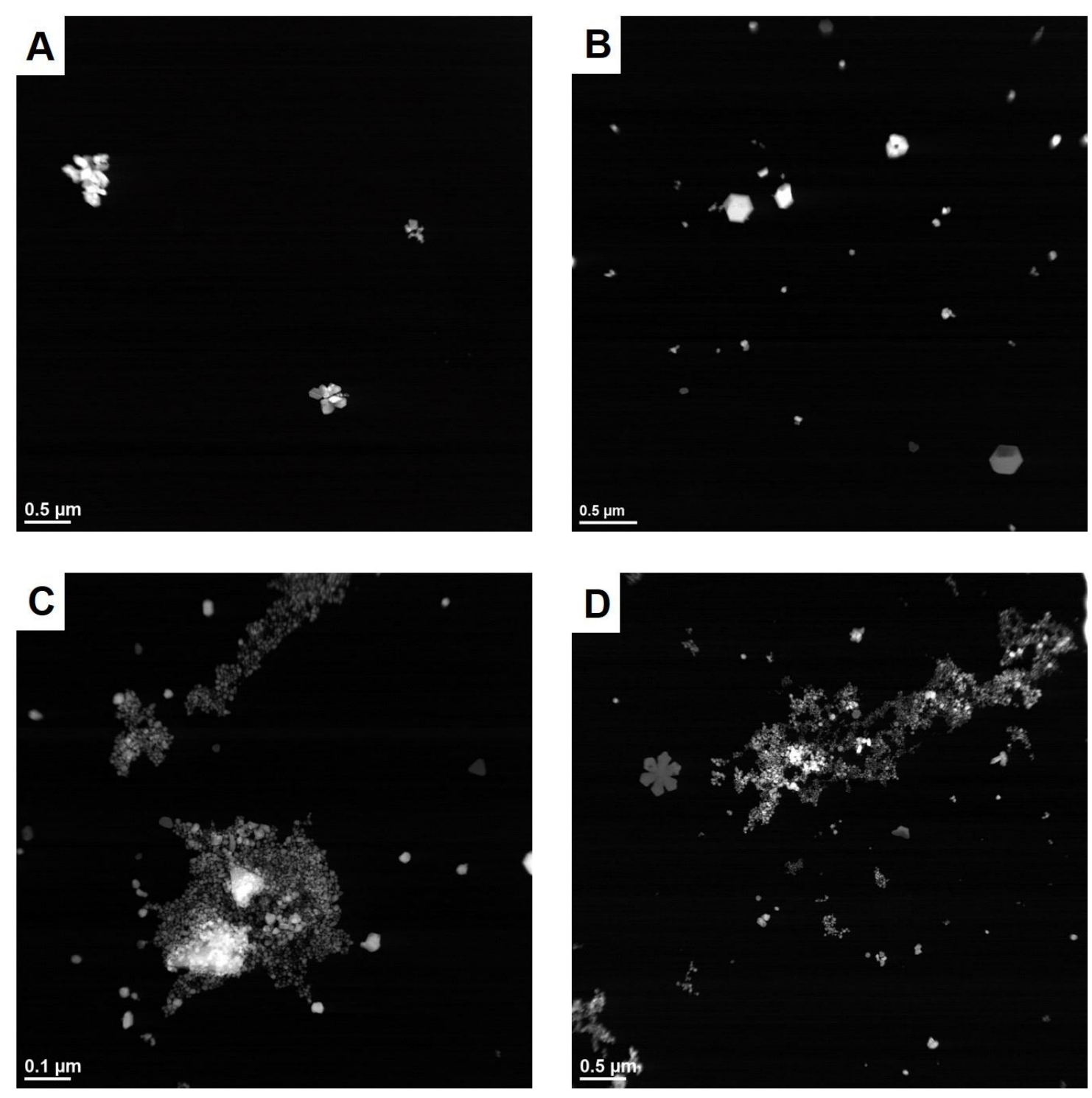

Figure S3. Representative STEM images of AgNPs formed in aqueous solutions of (A) 9 wt $\%$ glucose, (B) $9 \mathrm{wt} \%$ Domino table sugar, and (C) $0.45 \mathrm{wt} \%$ maltodextrin, and (D) white cranberry juice, with $\mathrm{Ag}^{+}$ concentration at $5.8 \mathrm{mM}$ after 3 hours introduction. AgNPs formed in maltodextrin and white cranberry juice show aggregation. 


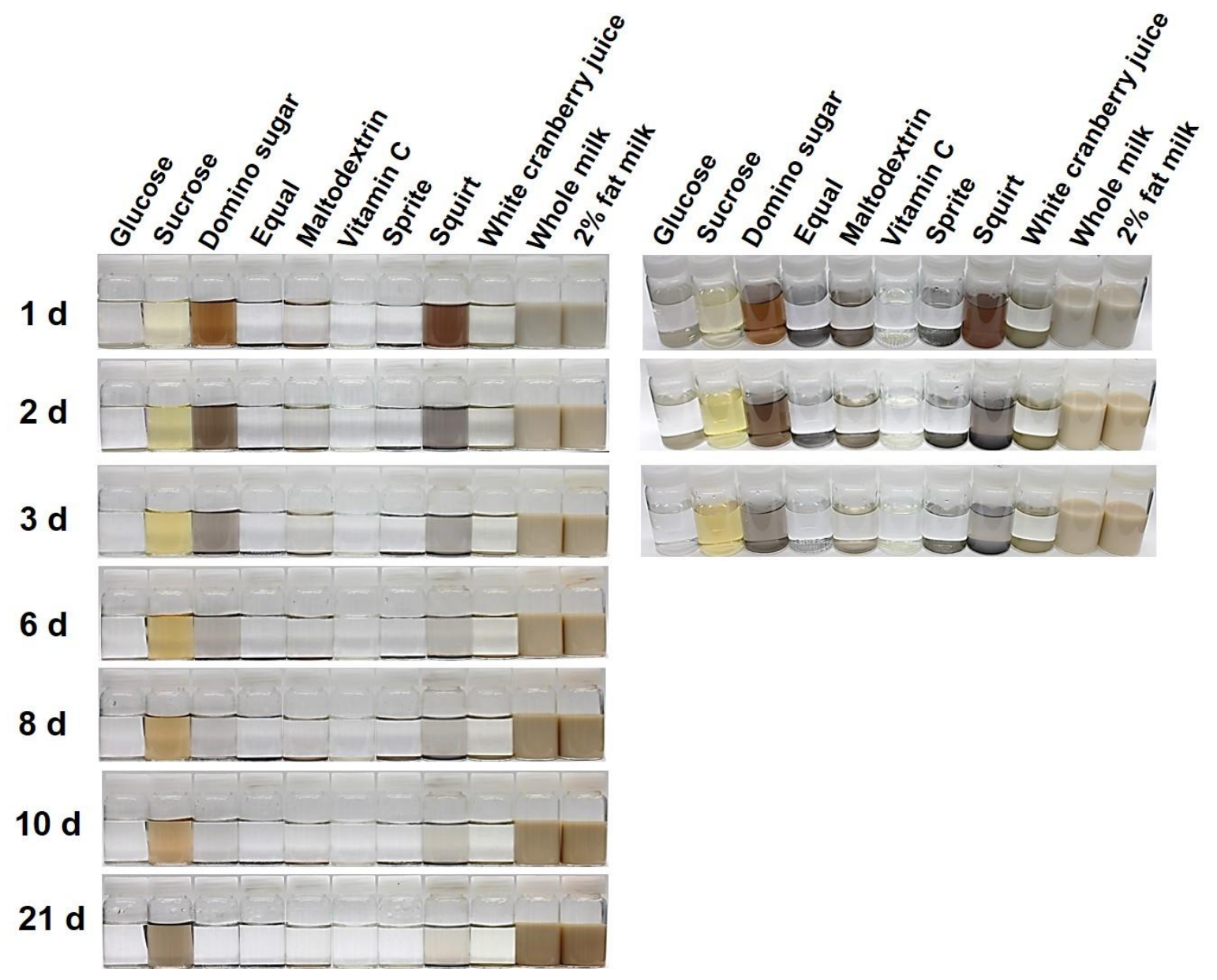

Figure S4. A series of photographs of AgNPs produced in aqueous solutions of natural and artificial sweeteners and commercial beverages after different time intervals. None of these samples were redispersed before photographing (compare to Figure 1, where samples were vortexed prior to photographing). The left panel shows a side on view. The right panel shows a top-down view for the first 3 days, in which aggregates of AgNPs are visible at the bottom of the vials. 


\section{Sprite}
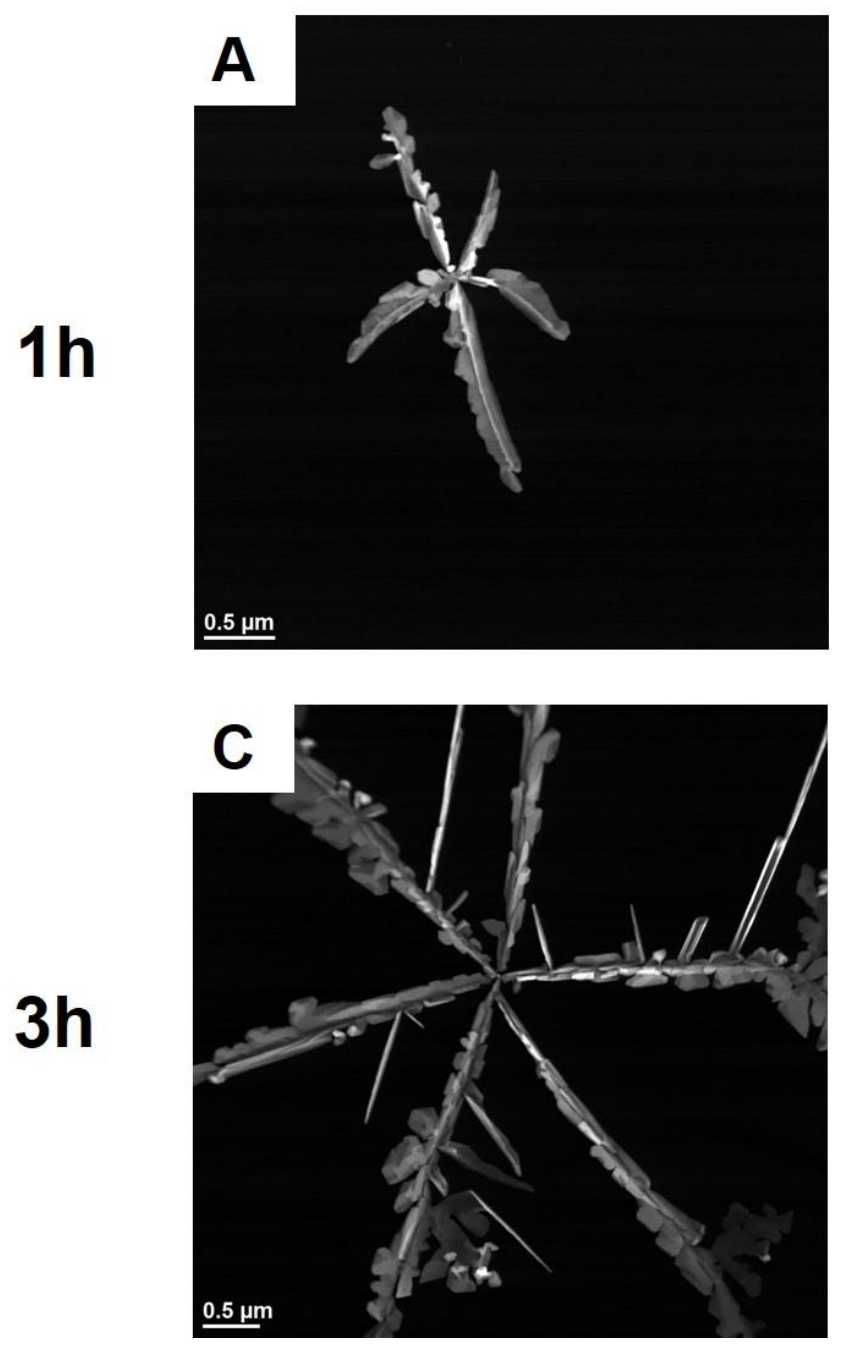

\section{Squirt}
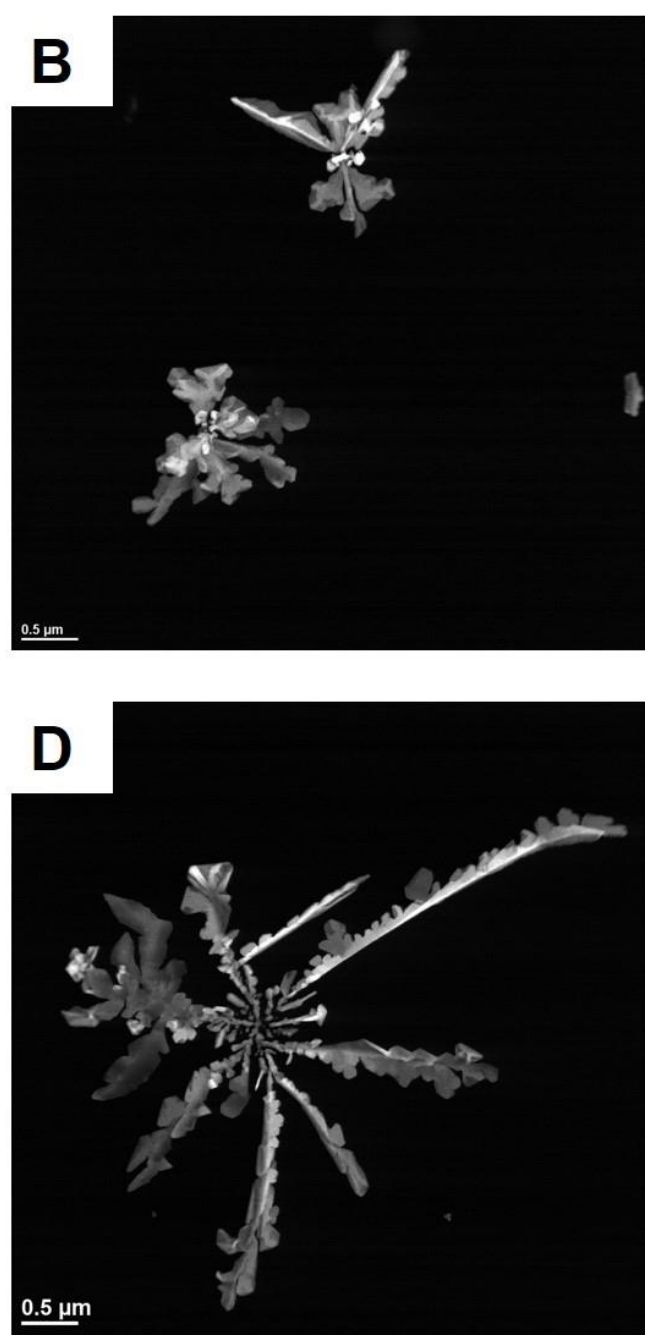

Figure S5. Representative STEM images of snowflake-like Ag structures formed in (A and C) Sprite and (B and D) Squirt drinks with $\mathrm{Ag}^{+}$concentration at $5.8 \mathrm{mM}$ after 1- and 3-hours introduction. 

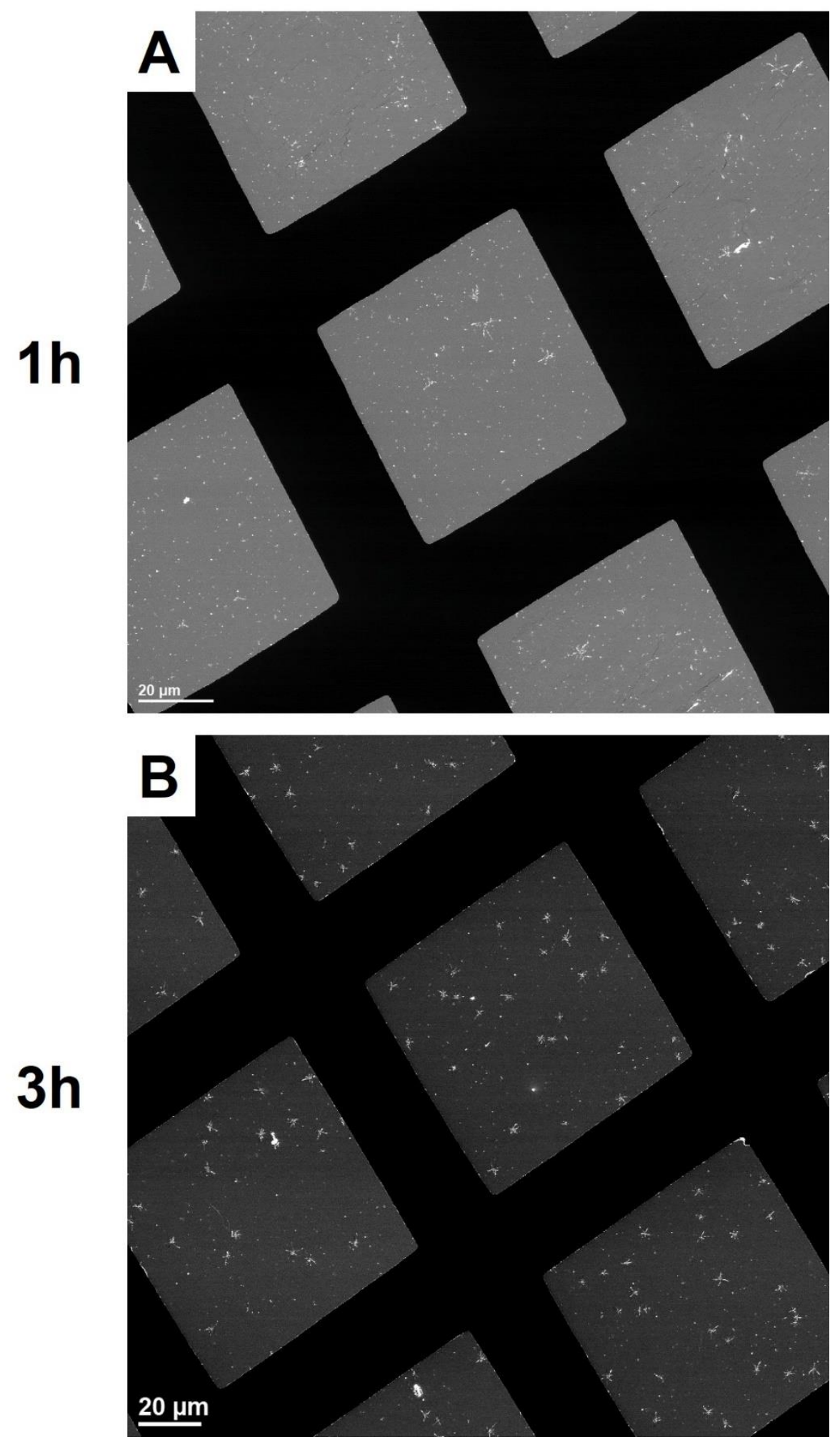

Figure S6. Representative overview of STEM images of snowflake-like Ag structures formed in (Squirt drinks with $\mathrm{Ag}^{+}$concentration at $5.8 \mathrm{mM}$ after (A) 1 and (B) 3-hours introduction. 

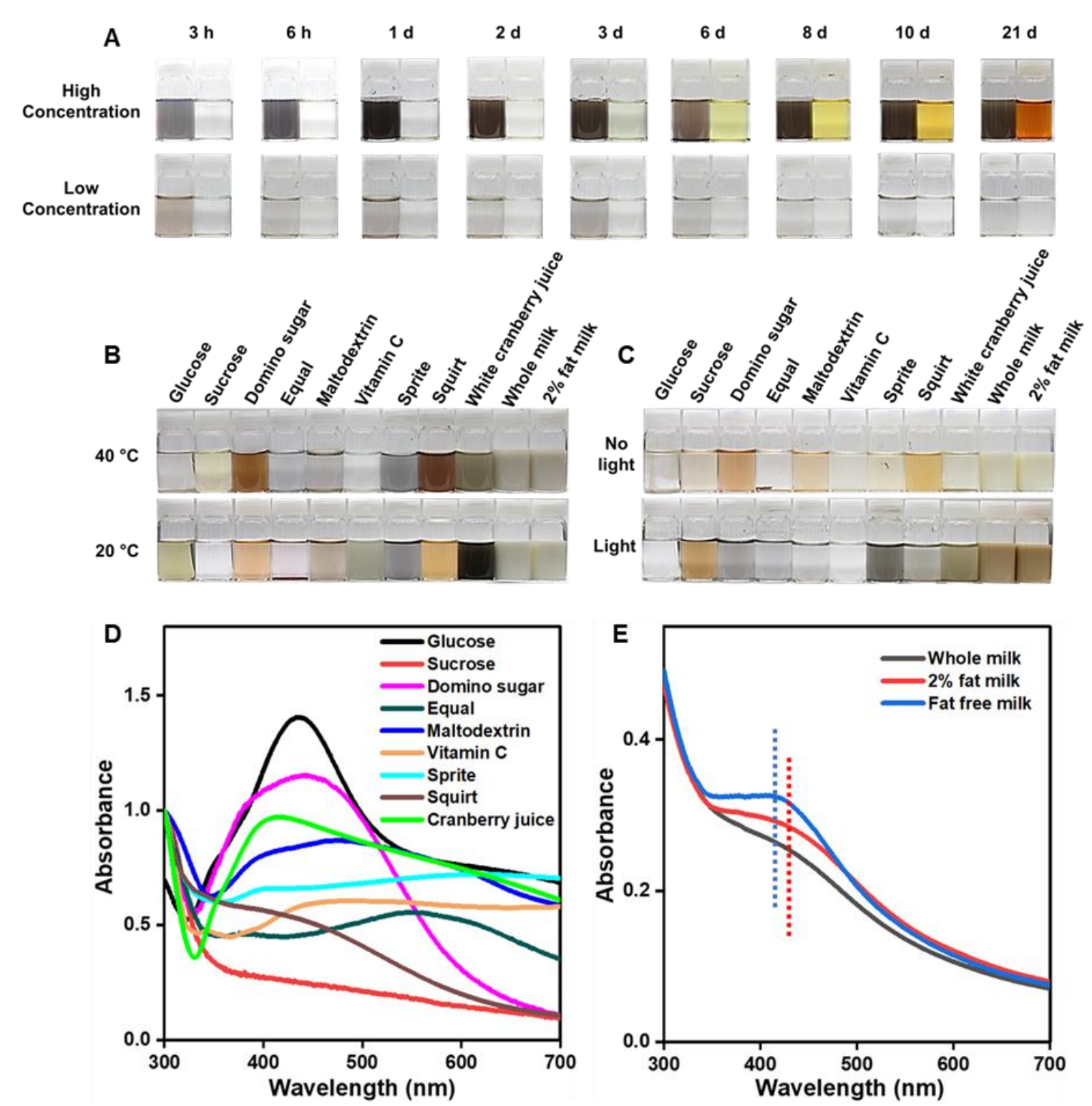

Figure S7. Effect of (A) reducing ingredient concentration, (B) storage temperature, and (C) light exposure on formation of AgNPs in aqueous solutions of food ingredients and commercial beverages after spiking with $3 \mathrm{~mL}$ of $25 \mathrm{mM}$ aqueous $\mathrm{AgNO}_{3}$. Panel (A) shows photographs of aqueous solutions of maltodextrin (left vial) and vitamin $\mathrm{C}$ (right vial) at two concentrations and acquired at different times after spiking with $\mathrm{AgNO}_{3}$ and storing at $40{ }^{\circ} \mathrm{C}$. In the high concentration group, both maltodextrin and vitamin $\mathrm{C}$ are at a concentration of $9 \mathrm{wt} \%$, and in the low concentration group, the concentrations of maltodextrin and vitamin $\mathrm{C}$ were $0.45 \mathrm{wt} \%$ and $0.03 \mathrm{wt} \%$, respectively. Panel (B) shows photographs of aqueous solutions of food ingredients and commercial beverages 24 hours after spiking with $\mathrm{AgNO}_{3}$ and storing at either $40{ }^{\circ} \mathrm{C}$ and $20{ }^{\circ} \mathrm{C}$. Panel (C) shows photographs of aqueous solutions of food ingredients and commercial beverages after spiking with $\mathrm{AgNO}_{3}$ and storing under ambient light (11 days) or in the dark (10 days). (D) Normalized UV-Visible spectra of AgNPs generated in aqueous solutions of food ingredients and commercial beverages after storage at $20^{\circ} \mathrm{C}$ for 24 hours post $\mathrm{AgNO}_{3}$ introduction. (E) Normalized UV-Visible spectra of AgNPs formed in whole fat, $2 \%$ fat, and fat free milk after storage at $20{ }^{\circ} \mathrm{C}$ for 3 days post $\mathrm{AgNO}_{3}$ introduction. The dashed blue and red line show SPR peaks of AgNPs in fat free milk and whole milk, respectively. 

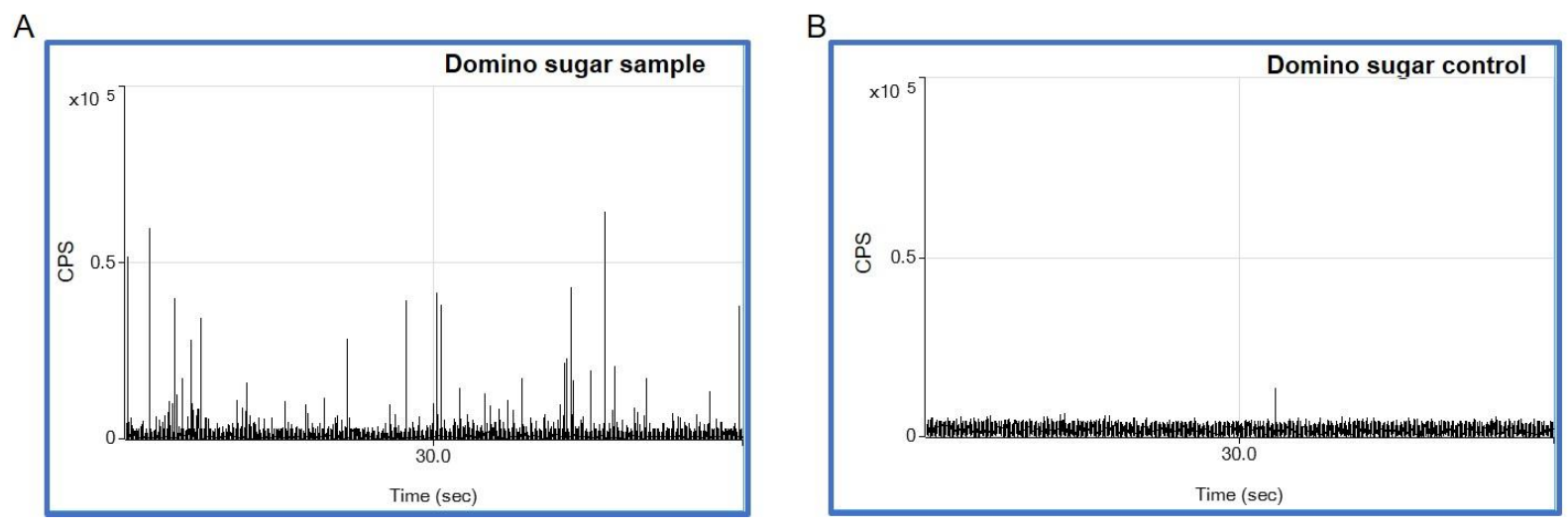

Figure S8. (A) SP-ICP-MS time scan of AgNPs present in aqueous Domino table sugar solutions with 0.25 $\mu \mathrm{g} / \mathrm{L} \mathrm{AgNO}_{3}$ stored for 10 days at $40{ }^{\circ} \mathrm{C}$. (B) Analogous time scan of the Domino sugar solution control (no $\mathrm{AgNO}_{3}$ added). Formed $\mathrm{AgNP}$ quantity is determined according to the number of spikes present during time scanning of a sample and the size of the spike correlates to the physical AgNP size.

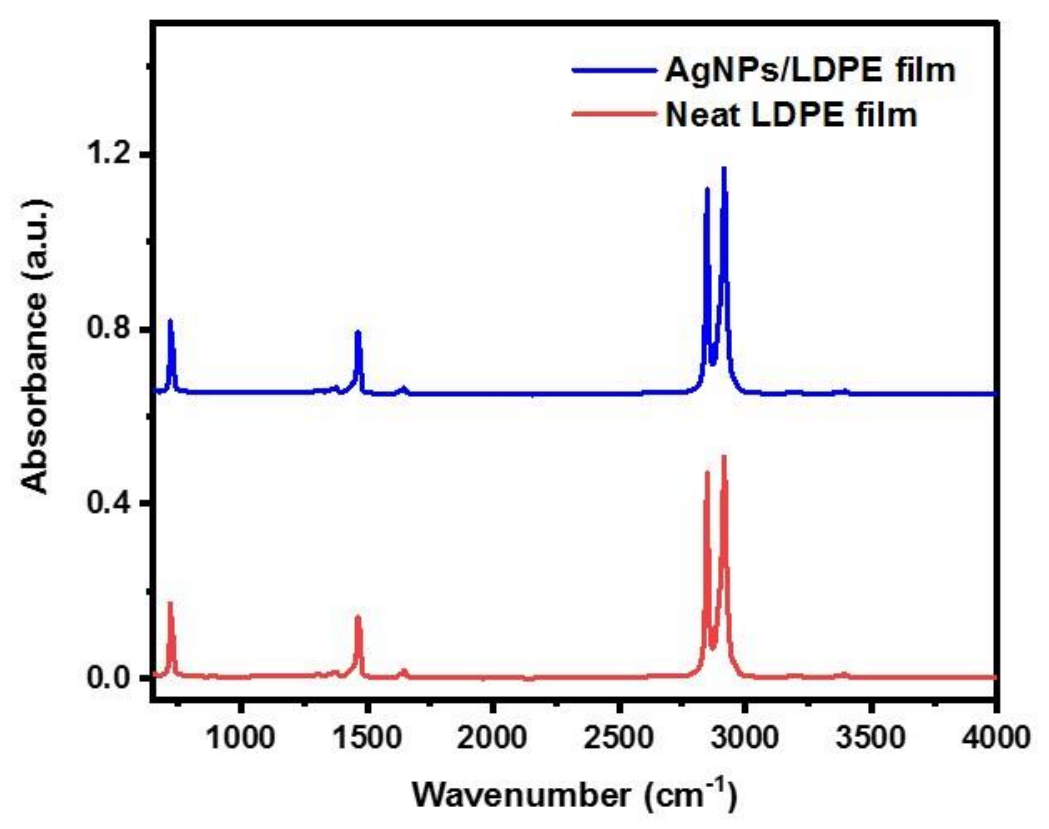

Figure S9. FTIR-ATR characterization of AgNPs/LDPE film and neat LDPE film. 


\section{References}

1. Kimling, J.; Maier, M.; Okenve, B.; Kotaidis, V.; Ballot, H.; Plech, A., Turkevich Method for Gold Nanoparticle Synthesis Revisited. The Journal of Physical Chemistry B 2006, 110 (32), 15700-15707.

2. Rahman, A.; Kumar, S.; Bafana, A.; Lin, J.; Dahoumane, S. A.; Jeffryes, C., A Mechanistic View of the Light-Induced Synthesis of Silver Nanoparticles Using Extracellular Polymeric Substances of Chlamydomonas reinhardtii. Molecules 2019, 24 (19), 3506.

3. Mittelman, A. M.; Fortner, J. D.; Pennell, K. D., Effects of ultraviolet light on silver nanoparticle mobility and dissolution. Environmental Science: Nano 2015, 2 (6), 683-691.

4. Hou, W.-C.; Stuart, B.; Howes, R.; Zepp, R. G., Sunlight-Driven Reduction of Silver Ions by Natural Organic Matter: Formation and Transformation of Silver Nanoparticles. Environ. Sci. Technol. 2013, 47 (14), 7713-7721.

5. Yin, Y.; Liu, J.; Jiang, G., Sunlight-Induced Reduction of Ionic Ag and Au to Metallic Nanoparticles by Dissolved Organic Matter. ACS Nano 2012, 6 (9), 7910-7919.

6. Hannon, J. C.; Kerry, J. P.; Cruz-Romero, M.; Azlin-Hasim, S.; Morris, M.; Cummins, E., Migration assessment of silver from nanosilver spray coated low density polyethylene or polyester films into milk. Food Packag. Shelf Life 2018, 15, 144-150.

7. Bayani Bandpey, N.; Aroujalian, A.; Raisi, A.; Fazel, S., Surface coating of silver nanoparticles on polyethylene for fabrication of antimicrobial milk packaging films. Int. J. Dairy Technol. 2017, 70 (2), 204-211.

8. Kumar, A.; Vemula, P. K.; Ajayan, P. M.; John, G., Silver-nanoparticle-embedded antimicrobial paints based on vegetable oil. Nature Mater. 2008, 7 (3), 236-241.

9. Kittler, S.; Greulich, C.; Diendorf, J.; Köller, M.; Epple, M., Toxicity of Silver Nanoparticles Increases during Storage Because of Slow Dissolution under Release of Silver Ions. Chem. Mater. 2010, 22 (16), 4548-4554.

10. Singh, H.; Gallier, S., Nature's complex emulsion: The fat globules of milk. Food Hydrocolloids 2017, 68, 81-89.

11. Bothun, G. D., Hydrophobic silver nanoparticles trapped in lipid bilayers: Size distribution, bilayer phase behavior, and optical properties. J. Nanobiotechnol. 2008, 6 (1), 13.

12. Zielińska-Jurek, A.; Reszczyńska, J.; Grabowska, E.; Zaleska, A., Nanoparticles preparation using microemulsion systems. Microemulsions-An Introduction to Properties and Applications 2012, 229250.

13. Bora, A.; Mishra, P., Casein and Ag nanoparticles: Synthesis, characterization, and their application in biopolymer-based bilayer film. J. Food Processing Preserv. 2019, 43 (9).

14. Bora, A.; Mishra, P., Characterization of casein and casein-silver conjugated nanoparticle containing multifunctional (pectin-sodium alginate/casein) bilayer film. J. Food Sci. Technol. 2016, 53 (10), 3704-3714. 